\title{
An Agent-Based Model of Leader Emergence and Leadership Perception within a Collective
}

\author{
Shun Cao $\mathbb{D}^{1,2}$ Neil G. MacLaren,,3 ${ }^{1,3}$ Yding Cao, ${ }^{1,2}$ Yingjun Dong, ${ }^{1,2}$ Hiroki Sayama $\mathbb{D}^{1,2,4}$ \\ Francis J. Yammarino, ${ }^{1,3}$ Shelley D. Dionne, ${ }^{1,3}$ Michael D. Mumford, ${ }^{5}$ Shane Connelly, ${ }^{5}$ \\ Robert Martin, ${ }^{5}$ Colleen J. Standish, ${ }^{5}$ Tanner R. Newbold, ${ }^{5}$ Samantha England, \\ and Gregory A. Ruark ${ }^{6}$ \\ ${ }^{1}$ Center for Collective Dynamics of Complex Systems, Binghamton University, State University of New York, Binghamton, \\ NY 13902-6000, USA \\ ${ }^{2}$ Department of Systems Science and Industrial Engineering, Binghamton University, State University of New York, Binghamton, \\ NY 13902-6000, USA \\ ${ }^{3}$ Bernard M. \& Ruth R. Bass Center for Leadership Studies, School of Management, Binghamton University, \\ State University of New York, Binghamton, NY, USA \\ ${ }^{4}$ Waseda Innovation Lab, Waseda University, Shinjuku, Tokyo 169-8050, Japan \\ ${ }^{5}$ Department of Psychology, University of Oklahoma, Norman, OK, USA \\ ${ }^{6}$ U.S. Army Research Institute for the Behavioral and Social Sciences, Fort Belvoir, VA, USA
}

Correspondence should be addressed to Shun Cao; scao12@binghamton.edu

Received 7 November 2019; Revised 15 January 2020; Accepted 3 February 2020; Published 28 April 2020

Academic Editor: José Manuel Galán

Copyright (c) 2020 Shun Cao et al. This is an open access article distributed under the Creative Commons Attribution License, which permits unrestricted use, distribution, and reproduction in any medium, provided the original work is properly cited.

Effective teamwork in an initially leaderless group requires a high level of collective leadership emerging from dynamic interactions among group members. Leader emergence is a crucial topic in collective leadership, yet it is challenging to investigate as the problem context is typically highly complex and dynamic. Here, we explore leadership emergence and leadership perception by means of computational simulations whose assumptions and parameters were informed by empirical research and humansubject experiments. Our agent-based model describes the process of group planning. Each agent is assigned with three key attributes: talkativeness, intelligence, and credibility. An agent can propose a suggestion to modify the group plan as a speaker or respond and evaluate others' suggestions and leadership as a listener. Simulation results suggested that agents with high values of talkativeness, intelligence, and credibility tended to be perceived as leaders by their peers. Results also showed that talkativeness may be the most significant and instantaneous predictor for leader emergence of the three investigated attributes: talkativeness, intelligence, and credibility. In terms of group performance, smaller groups may outperform larger groups regarding their problem-solving ability in the beginning, but their performance tends to be of no significant difference in a long run. These results match the empirical literature and offer a mechanistic, operationalized description of the collective leadership processes.

\section{Introduction}

Traditional leadership assumes a single, often heroic, leader in a group or team. In contrast, collective leadership describes a process in which multiple individuals interacting with each other in an initially leaderless group or team can spontaneously direct their decision-making process, make decisions, and take actions on their own [1]. Multiple members within the group might serve as leaders in formal or informal capacities, and the shifting of leadership responsibilities is often rooted in individuals' characteristics, skills, and expertise [2].

Research on collective leadership has recently attracted significant attention as it has increasingly been recognized as a key role in group planning in rapidly changing complex environments [3-7]. The dynamics of leader emergence is a 
complex process contributed to by many correlated factors. Participation time (i.e., the amount of speaking time) is closely related to observer perceptions of leader emergence [8]. Several studies in psychology and sociology can bolster this statement $[9,10]$. Our own experimental study also finds a high correlation between speaking time and leader emergence as well [11]. Intelligence is typically considered to be a significant predictor of leader emergence in general $[12,13]$. It is known to be correlated positively and significantly, but not substantially, with leader emergence [14]. Finally, credibility is considered a cornerstone of leadership, which can significantly and positively impact leader emergence [15]. At a collective level, the group size is highly correlated with leader emergence. The average participation time is shorter in larger groups [16], where the distribution of participation time is more skewed toward a small number of participants. It was also reported that the larger the group is, the more inhibited introverts are [17], which supports this point of view. Increasing group size also makes the correlation between leadership perception and participation time stronger [18]. Meanwhile, group performance in a problemsolving context is argued to be independent of the group size $[18,20]$.

Long in the purview of researchers, leader emergence and leadership perception have been well studied. However, little research has been carried out regarding the development of a mechanistic theoretical framework that addresses leader emergence and leadership perception, not to mention operationalized computational models of it. To address the lack of mechanistic understanding of collective leadership, we construct a computational agent-based model of leader emergence from an initially leaderless group. We emphasize that our model is not developed to discover novel outcome behaviors or to detect explicit linkages between parameter and outcomes, but rather, to connect all of them and explain the process of leadership perception and leader emergence using a mechanistic means. The model is based on the theory of shared mental model formation in the teamwork and leadership literatures [7, 21-23]. Specifically, we quantitatively capture leader emergence by dynamical changes of a variable called perceived leadership, i.e., perception of an agent's leadership by others in a simulated group planning process. We also measure participation time and group performance under multiple group sizes. Our simulation results match findings and observations reported in the empirical literature: (1) leadership perception is highly correlated with the agent's talkativeness, intelligence, and credibility, and talkativeness may be the most significant and instantaneous predictor for leader emergence; (2) increasing group size lowers the average participation time but makes the correlation between participation time and leadership perception stronger; and (3) in the context of problemsolving ability, smaller groups outperform larger groups in the beginning of group planning, but they tend to be of no significant difference in the long term.

The rest of this paper is structured as follows. Details of our agent-based model, including attributes of agents and procedures of simulation, are introduced in Section 2. Simulation results regarding leader emergence, participation time, and group performance are shown in Section 3. Finally, conclusions are provided in Section 4.

\section{Agent-Based Model}

Agent-based models are widely used to model dynamical behaviors of systems in a variety of fields, such as economics, social science, organizational science, behavioral ecology, and physics. The essential idea is that simple behavioral rules of agents at microscopic scales may generate complex nontrivial behaviors at macroscopic scales [24]. We set the collective leadership scenario in the context of a small-sized group of agents trying to solve an optimization problem in an unknown problem space through group discussion for a finite period. The group size is in the range of $4 \sim 10$, since very small groups (i.e., 2 or 3 agents) lack diversity of perspectives, whereas larger groups tend to split into subgroups [25,26]. The agents are assumed to be in a fully connected social network, to possess equal social status, and to have individual attributes that are different from each other. The model settings are mainly based on our earlier work [27], with significant revisions added to capture the collective leadership at individual and group levels. The present model has implemented a procedure for leadership evaluation, modified individual utility functions, and improved each agent's local search strategy according to our new research objective. A schematic illustration of our new model is shown in Figure 1.

2.1. Problem Space. The optimization problem is defined on a continuous space $S=[0,1]^{m}$, where $m$ represents the dimensions of the problem space which are also called the aspects of the problem. The true utility function is defined in this problem space but is not accessible from any of the agents. A plan is defined as a point (i.e., $m$-dimensional vector) in the problem space. An opinion of an agent is a plan with a utility calculated by its individual utility function. The distance between two plans or between a plan and an opinion means the distance of two points in the problem space. The true utility function is defined as follows:

$$
\begin{aligned}
U(v)= & \sum_{i=1}^{m} \sum_{k=1}^{l} E_{i k}^{L} \sin \left(\omega_{i k} x_{i}\right) \\
& +\sum_{\substack{1 \leq i, j \leq m \\
i \neq j}} \sum_{k=1}^{l} E_{i j}^{I} \sin \left(\omega_{i k} x_{i}\right) \sin \left(\omega_{j k} x_{j}\right) .
\end{aligned}
$$

Here, $v=\left(x_{1}, x_{2}, \ldots, x_{m}\right)$ is a plan in the problem space and $l$ is the number of coexisting frequencies in each dimension. Those frequencies are randomly generated from a certain range so that $\omega_{i j} \in[0,10] . E_{i k}^{L}$ and $E_{i j}^{I}$ are the linear and interaction coefficients, respectively, which are generated from the random value in the range so that $E \in[0,1]$. This produces a bumpy, highly nonlinear utility function whose optimum is not easily discovered by agents (Figure 1, top left). 


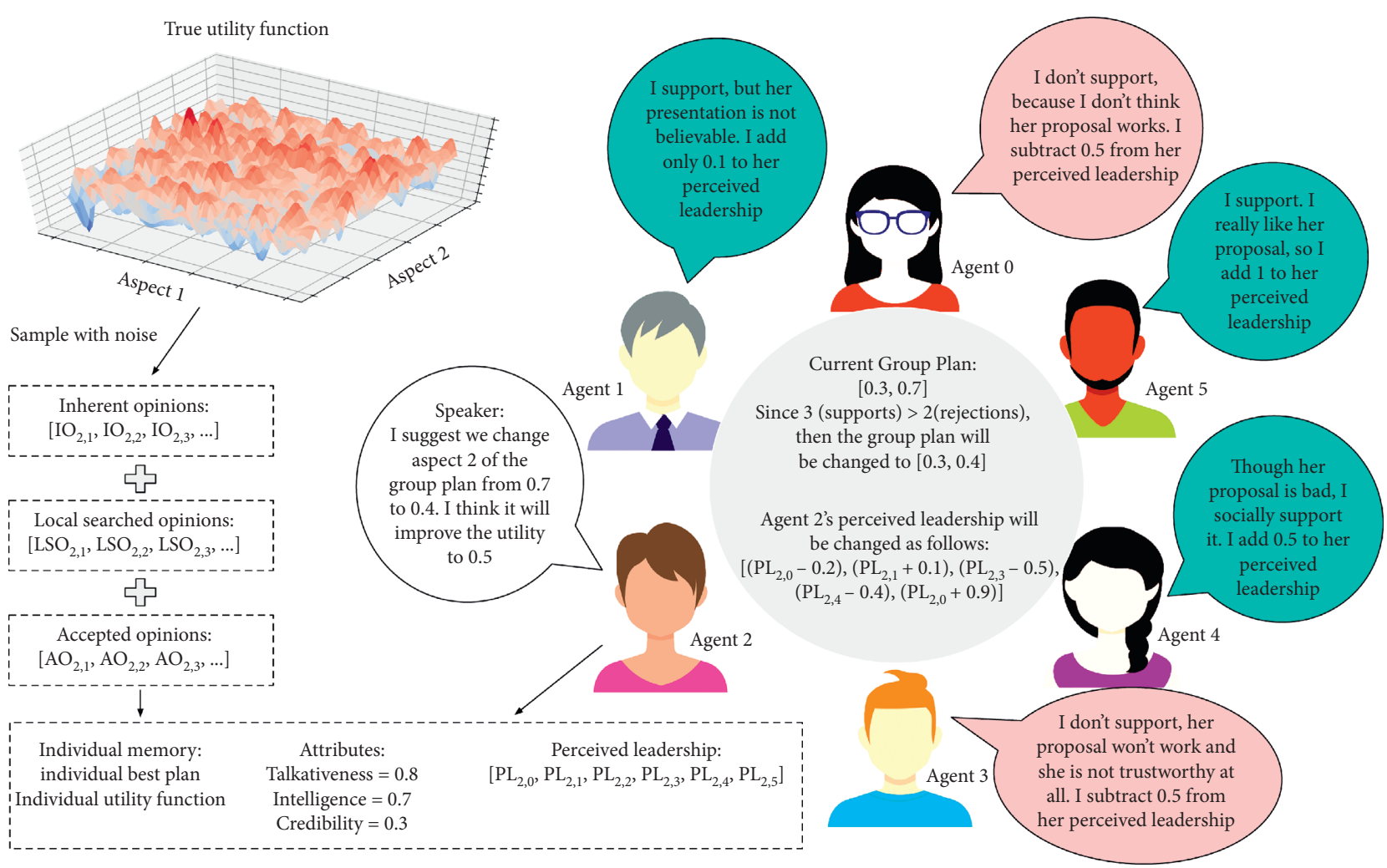

FIGURE 1: A schematic illustration of our agent-based model. A leaderless group consisting of $n$ agents trying to find the maximum point of the true utility function displayed in the top-left corner, which is not directly accessible from any agent. Agents have limited knowledge about the true utility function. Prior to discussion, each agent initially samples a certain number of opinions from the true utility function as its inherent opinions, whose quantity and quality depend on its intelligence. Agents reconstruct models of the utility function as their individual utility functions from those memorized opinions. Meanwhile, each agent also picks one plan with highest utility in its memory as its current best plan. The individual utility function and the best plan will be updated as new opinions are added to memory. In the planning process, the group members either keep silent and think about the problem by themselves or discuss about the problem openly. When silent, each agent studies a new opinion around its best plan by itself, then updates its individual utility function. Also, it changes its best plan if the new opinion is better. The speaking process goes as follows. First, a speaker proposes a suggestion of modifying one aspect of the group plan. Second, other agents (listeners) evaluate the speaker's perceived leadership according to the quality of its suggestion and the performance of its presentation. Finally, the listeners decide whether to adopt the speaker's suggestion or not at individual and group levels. If the new plan is supported by more than half of the listeners, the group plan will be changed according to the speaker's suggestion, otherwise the group plan remains unchanged. This cycle repeats for a certain number of iterations.

2.2. Agents. Based on our literature review, we assign the following three key attributes to each agent: (1) talkativeness, (2) intelligence, and (3) credibility. For simplicity, we assumed these attributes are distributed within the range of $[0,1]$. We also endowed each agent with a list of perceived leadership attributes (i.e., cumulative leadership perception given to it by peers) and an individual memory including an individual utility function, a set of memorized opinions, and its current best plan. Each agent will update its best plan and individual utility function as more opinions are added to its memory. More details of these model components are described below:

Talkativeness refers to the willingness of proposing a new suggestion in the group planning process. Those with higher talkativeness tend to act more often as a speaker. We use $T_{i}$ to indicate agent $i$ 's talkativeness. Intelligence is a general ability that correlates with multiple intellectual skills, behaviors, and outcomes. In our model, it decides the quality and quantity of each agent's inherent opinions and its local search radius. We use $I_{i}$ to indicate agent $i$ 's intelligence.

Credibility relates to the gain of leadership perception. If the speaker's credibility is high, the listeners are more inclined to give a high evaluation of the speaker's leadership and support its suggestion. We use $C_{i}$ to represent agent $i$ 's credibility.

Inherent opinions are opinions each agent initially samples from the true utility function. The number of agent $i$ 's inherent opinions is $\varphi I_{i}+2$, where $I_{i}$ is agent $i$ 's intelligence and $\varphi$ is a parameter. Inclusion of 2 guarantees that each agent will have at least two opinions to construct its individual utility function $(k$ NN) initially. The utility of agent $i$ 's inherent opinion is its true utility perturbed by random noise $\delta \in\left[I_{i}-1,1-I_{i}\right]$. Note the noise range narrows toward zero as agent $i$ 's intelligence approaches 1 . 
Individual opinions are all opinions memorized in an agent's memory, which consist of three parts: inherent opinions $\left\{I O_{i, 1}, I O_{i, 2} \ldots\right\}$, locally searched opinions $\left\{L S O_{i, 1}, L S O_{i, 2} \ldots\right\}$, and opinions accepted from other agents $\left\{A O_{i, 1}, A O_{i, 2} \ldots\right\}$ (Figure 1). Agent $i$ 's individual opinion set is represented as $O_{i}=\left\{I O_{i, 1}, I O_{i}\right.$, $2 \ldots\} \cup\left\{L S O_{i, 1}, L S O_{i, 2} \ldots\right\} \cup\left\{A O_{i, 1}, A O_{i, 2} \ldots\right\}$.

Individual best plan is the plan with the highest utility in an agent's memory based on its individual utility function. Agent $i$ 's best plan is represented as $v_{i \text {,best }}$.

Individual utility function is an agent's internal function that maps a plan to a utility value. This function estimates the utility of a plan through $k$-nearest neighbor regression $(k-\mathrm{NN})$ [28] with the agent's individual opinions used as the neighbor dataset. Here, we assume each agent can evaluate all the proposed plans.

Perceived leadership is the variable that captures leader emergence that dynamically changes according to speaking times, agent's credibility, and the quality of its proposed plans. Agent $i$ 's perceived leadership is represented as a vector $P L_{i}=\left(P L_{i, 1}, P L_{i, 2}, \ldots, P L_{i, n}\right)$, where $P L_{i, j}$ represents the perceived leadership given to agent $i$ by another agent (listener) $j$. Note that $P L_{i}$ does not contain self-evaluation $\left(P L_{i, i}\right)$.

2.3. Group Planning. In the group planning process, agents either talk about the problem openly or keep silent and think about the problem by themselves. The ratio between silence and speaking varies according to group size. It was empirically reported that the average speaking time was $47 \%$ in two-person groups and $29 \%$ in six-person groups [16]. We used these numbers to construct a simple linear model of the speaking time ratio as a function of group size $n$ : Speaking Time Ratio $=a n+b$, with $(a, b)=(-0.045,0.56)$. In our model, this ratio was used as a probability of speaking by any agent in each iteration.

In the silence state, every agent keeps silent and independently examines whether there is a better plan around its best plan to achieve a higher utility. Specifically, each agent randomly selects and memorizes a new plan from a neighborhood of its best plan, $\left\{v \mid\left\|v-v_{i \text {,best }}\right\|<r\right\}$, where $r$ is the local search radius. The agent will update its best plan if the new plan is better.

In the speaking state, there are five steps that make up a speaking cycle, which is shown in Figure 2. These five steps are described below:

Step 1: selection of a speaker.

One agent is selected probabilistically from all agents based on their talkativeness. The speaking probability of agent $i$ is calculated as $p_{i}=T_{i}^{\xi} / \sum_{j=1}^{n} T_{j}^{\xi}$, where $\xi \geq 1$ is an adjustable exponent. When $\xi=1$, selection probabilities of a speaker are proportional to the agents' talkativeness. Increasing $\xi$ will amplify the impact of talkativeness in the selection of the speaker.

Step 2: proposal of a new plan.

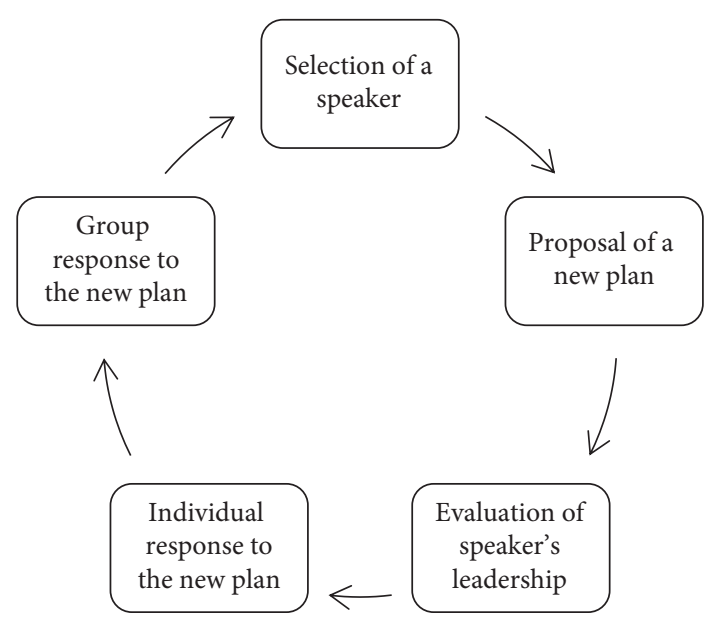

FIGURE 2: A speaking cycle made of five steps of group planning used in our model.

The selected speaker generates $m$ new plans by replacing each of the $m$ aspects of the current group plan with the corresponding value in its individual best plan. Then, the speaker chooses the plan with the highest utility and proposes it to the other agents.

Step 3: evaluation of speaker's leadership.

After the speaker $i$ proposes a new plan $v_{\text {new }}$ and the corresponding utility $U_{i}\left(v_{\text {new }}\right)$, every listener evaluates the speaker's leadership. Listener agent $j$ evaluates the utility of the new plan using its own utility function $\left(U_{j}\left(v_{\text {new }}\right)\right)$.

If $\left|U_{j}\left(v_{\text {new }}\right)-U_{i}\left(v_{\text {new }}\right)\right|<\tau e^{-\alpha(t / h)}$ (where $\tau, \alpha, h$, and $t$ are the initial tolerance, a constant in time decay term, the total number of iterations, and the current number of iterations, respectively [27]), agent $i$ 's perceived leadership from agent $j$ 's perspective will increase as $P L_{i, j} \leftarrow P L_{i, j}+\beta C_{i} n e^{-\alpha(t / h)}+\varepsilon$, where $C_{i}$ is agent $i$ 's credibility, $n$ is the group size, and $\varepsilon$ is a constant reward for speaking. We included the group size here to capture the empirical observation that the group size can affect the gain of leadership perception. Namely, a speaker communicating to large audience may gain greater perceived leadership than a speaker talking to just a few listeners [18]. The purpose of using the decay term $e^{-\alpha(t / h)}$ is to represent temporal decay of tolerance to bad ideas and credibility's contribution to speaker's leadership perception.

In the opposite scenario, if $\left|U_{j}\left(v_{\text {new }}\right)-U_{i}\left(v_{\text {new }}\right)\right| \geq$ $\tau e^{-\alpha(t / h)}$, the perceived leadership will be updated as $P L_{i, j} \leftarrow P L_{i, j}+\beta C_{i} n e^{-\alpha(t / h)}-\gamma e^{-\alpha(t / h)}$, where the last negative term represents a penalty incurred on the speaker's leadership perception when its judgement of the new plan is too far away from the listener's estimation. Such a rejection situation arises more frequently as time proceeds because the tolerance level $\tau e^{-\alpha(t / h)}$ decreases exponentially. To avoid speaker agents overpenalized in the later stage of simulation, the penalty term is also scaled with $e^{-\alpha(t / h)}$. 
Step 4: individual response to the new plan.

Each listener individually decides whether it accepts or rejects the new plan proposed by the speaker. If $\left|U_{j}\left(v_{\text {new }}\right)-U_{i}\left(v_{\text {new }}\right)\right|<\tau e^{-\alpha(t / h)}$, the listener will accept and add the new plan to its memory. Otherwise, the listener will not add the new plan to its memory. However, the listener may still express social acceptance [14] for the new plan. The probability for listener $j$ 's social acceptance of speaker $i$ 's new plan is given by $S A_{i}=d_{i}^{\min } C_{i} e^{-\alpha(t / h)}$, where $d_{i}^{\text {min }}$ is the minimum of the Euclidean distances among agent $i$ 's memorized plans and the new plan. When $d_{i}^{\mathrm{min}}$ is large, that means the new plan is far from the knowledge of the listener, so it tends to increase the probability of social acceptance. Larger credibility of the speaker will also increase the probability of social acceptance. The time-dependent decay is also included to decrease the probability of social acceptance as the discussion continues.

Step 5: response to the suggestion in group level.

After all the listeners have made their acceptance/rejection decisions, the group as a whole responds to the new plan in a democratic manner. If the proposed plan is supported by more than half of the listeners, the new plan will be accepted as a new group plan. Otherwise, the group plan will remain unchanged.

\section{Simulation Results}

We implemented the simulation model in Python and ran the experiments in multiple scenarios. We use two group sizes as representative cases: 4-agent group and 8-agent group, whose attributes of agents are randomly generated. Details are shown in Tables 1 and 2, respectively. Note that these specific 4-agent and 8-agent settings were used only for the results shown in Figures 3-7.

To obtain the simulation results, the following parameter settings were commonly used (we have tested minor variations of these values and confirmed that the main results and conclusions were not significantly changed):

(i) Dimension of problem space: $m=2$

(ii) Number of coexisting frequencies in the true utility function per dimension: $l=5$

(iii) Parameter for determining the number of agent's inherent opinions: $\varphi=20$

(iv) Number of nearest neighbors used for calculating agent's individual utility function: $k=2$

(v) Radius of local search for each agent: $r=0.005$

(vi) Exponent for probability of speaking: $\xi=3$

(vii) Constant for temporal decay term: $\alpha=1.3$

(viii) Initial utility tolerance for evaluation of perceived leadership: $\tau=0.5$

(ix) Constant for evaluation of perceived leadership: $\beta=0.05$

(x) Reward and penalty for evaluation of perceived leadership: $\varepsilon=0.4$ and $\gamma=0.2$
TABle 1: Agents' attributes in a representative 4-agent group. Note that all attributes are normalized in the range of $[0,1]$.

\begin{tabular}{lccc}
\hline Agent & Talkativeness & Intelligence & Credibility \\
\hline Agent 0 & 0.51 & 0.50 & 0.81 \\
Agent 1 & 0.33 & 0.94 & 0.34 \\
Agent 2 & 0.62 & 0.87 & 0.68 \\
Agent 3 & 0.72 & 0.23 & 0.15 \\
\hline
\end{tabular}

TABle 2: Agents' attributes in a representative 8-agent group.

\begin{tabular}{lccc}
\hline Agent & Talkativeness & Intelligence & Credibility \\
\hline Agent 0 & 0.11 & 0.46 & 0.72 \\
Agent 1 & 0.91 & 0.95 & 0.84 \\
Agent 2 & 0.25 & 0.64 & 0.96 \\
Agent 3 & 0.49 & 0.22 & 0.98 \\
Agent 4 & 0.64 & 0.91 & 0.31 \\
Agent 5 & 0.39 & 0.45 & 0.48 \\
Agent 6 & 0.77 & 0.13 & 0.27 \\
Agent 7 & 0.52 & 0.88 & 0.45 \\
\hline
\end{tabular}

3.1. Leader Emergence. The first and most important problem we examined is leader emergence. We ran each simulation for 300 iterations. The results with the 4-agent group and the 8-agent group are displayed in Figures 3 and 4 , respectively. The top left panel of Figure 3 shows the dynamical change of each agent's perceived leadership. After 300 discussion rounds, agent 2 gained the highest perceived leadership from its peers. Agent 2 had relatively high values of talkativeness $\left(T_{2}=0.62\right)$, intelligence $\left(I_{2}=0.87\right)$, and credibility $\left(C_{2}=0.68\right)$, which helped it to be the leader, though none of these attributes were the highest in the group. At the beginning of discussion, agents lacked knowledge or information to judge the quality of new proposed plans, and they were more inclined to accept new plans as listeners. Therefore, the agents who talked more at first received more rewards than other less talkative ones. In this case, agent 3 , the most talkative $\left(T_{3}=0.72\right)$ agent in the group gained higher perceived leadership than agent 2 in the initial stage of the simulation. However, agent 3's intelligence and credibility were rather low $\left(I_{3}=0.23\right.$ and $\left.C_{3}=0.15\right)$, making its proposed new plans prone to large errors and questionable. Other agents might accept agent 3 's plans at the beginning, but as discussion and communication continued, other agents gained more information about the problem space, which enabled them to judge the quality of a new plan more reasonably. Then, agent 3's new plans became rejected more frequently for the rest of iterations, which is seen as its high rejection rate in the top right panel of Figure 3. Meanwhile, agent 2's plans were most often accepted by the group because of its high intelligence. For other members, agent 0 's credibility $\left(C_{0}=0.81\right)$ was the highest in the group, but its intelligence was not so high $\left(I_{0}=0.5\right)$ and most of its proposed plans were rejected by the group. Agent 1 was the least talkative $\left(T_{1}=0.33\right)$ agent, but it was very smart $\left(I_{1}=0.94\right)$ which made its only few proposals all adopted by the group. Both agent 0 and agent 1 had some shortcomings that prevented them from becoming the leader of the group. 


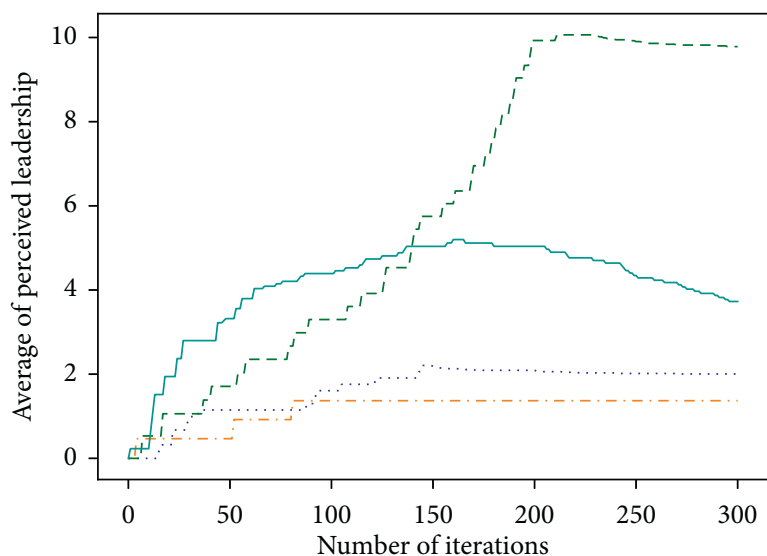

… Agent 0

.... Agent 1

- - - Agent 2

- Agent 3

(a)

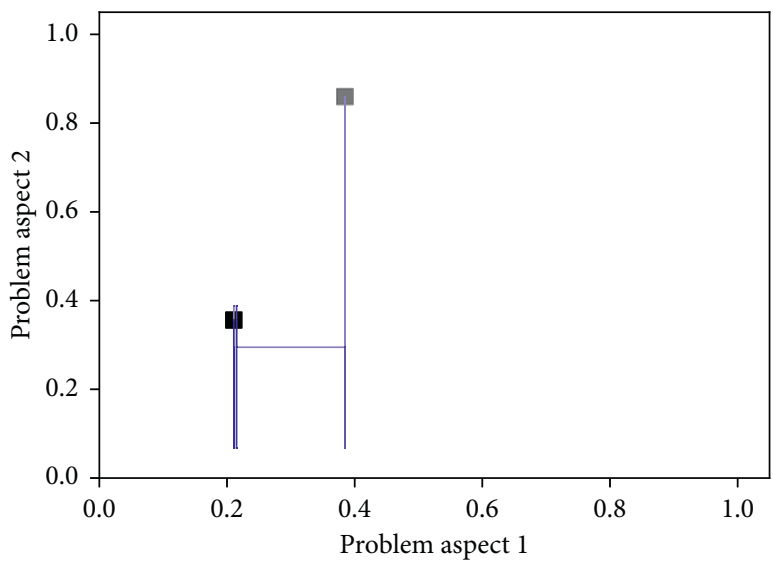

Initial group plan

Final group plan

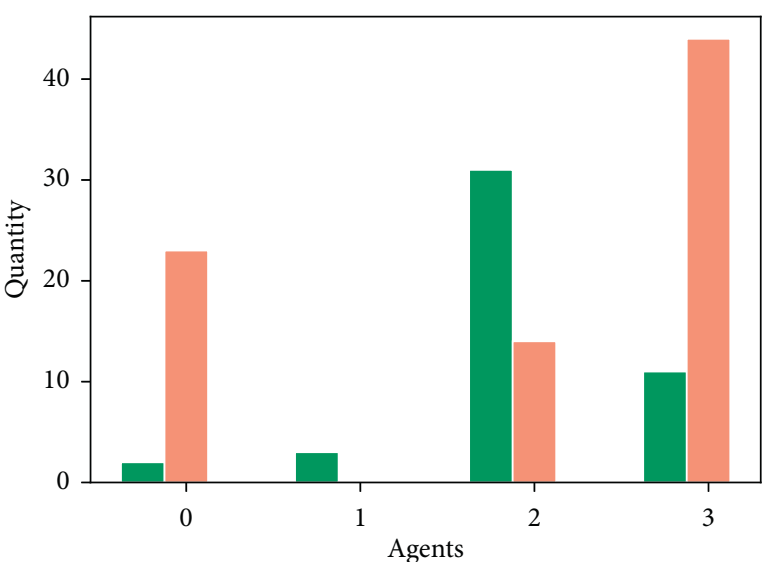

Accepted

Rejected

(b)

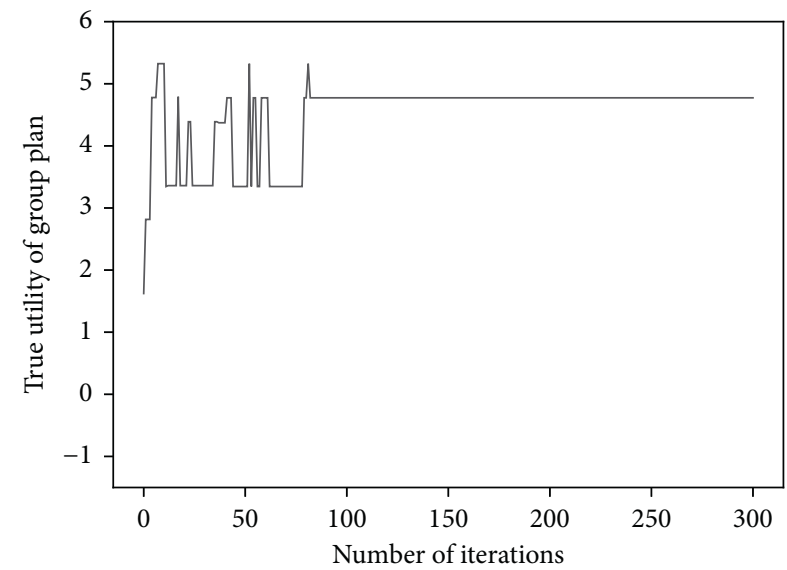

(d)

Figure 3: Simulation results of the representative 4-agent group. (a) Dynamical change of the agents' perceived leaderships. The average of each agent's perceived leadership is shown in this plot. (b) Quantities of adopted and rejected plans proposed by each agent. (c) The trajectory of the group plan. (d) The change of the group plan's true utility. Note that agents' attributes are defined in Table 1.
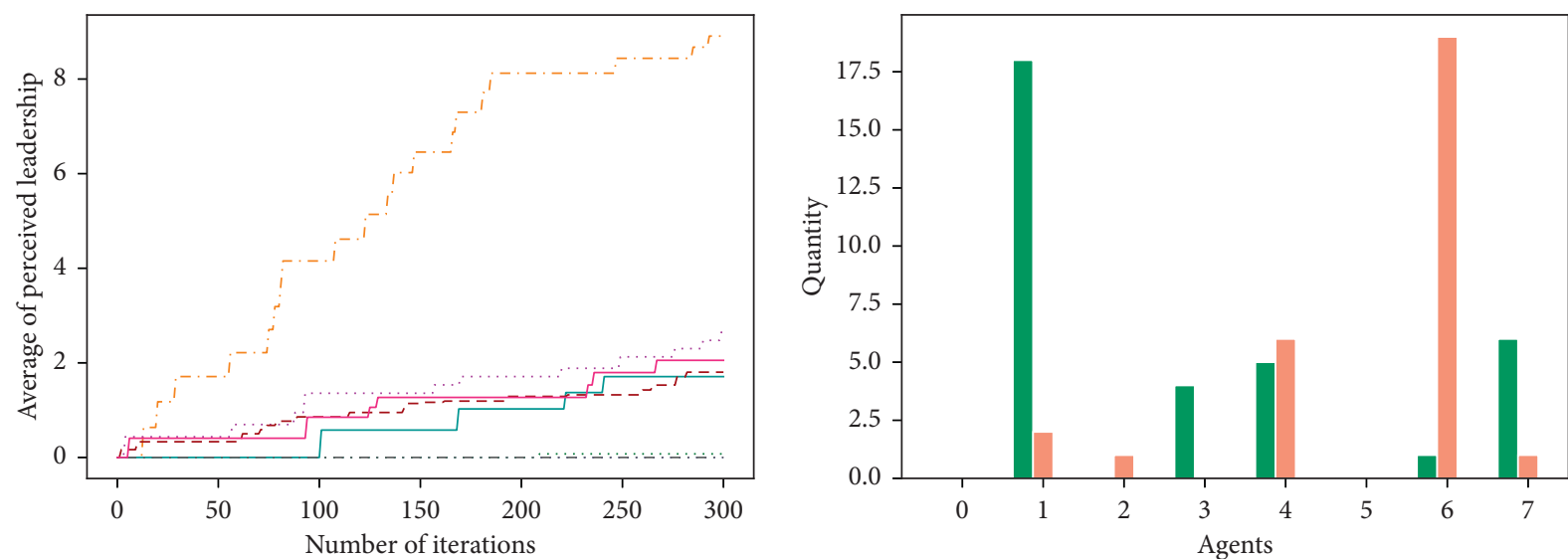

Agent 0

-. - Agent 1

Agent 2

…. Agent 4

-. - Agent 5

Accepted

Agent 3

- - - Agent 6

- Agent 7

(a)

Figure 4: Continued. 


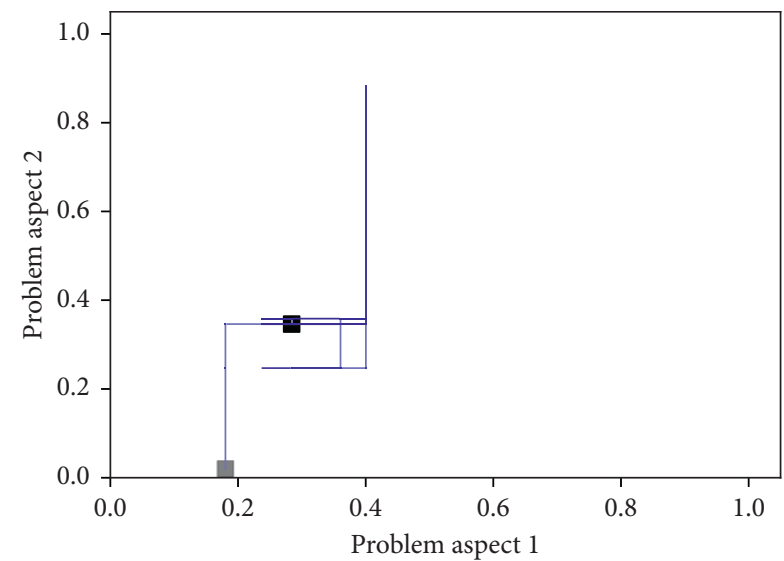

Initial group plan

- Final group plan

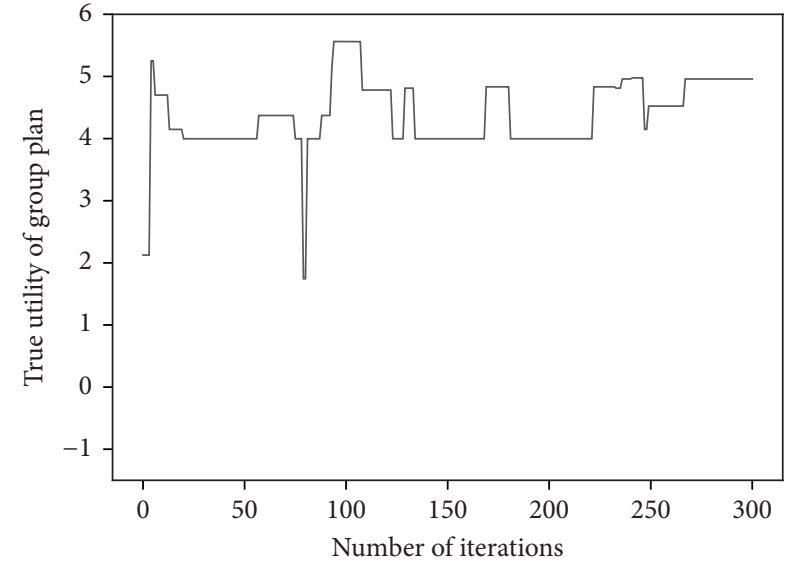

(d)

Figure 4: Simulation results of the representative 8-agent group. (a) Dynamical change of the agents' perceived leaderships. The average of each agent's perceived leadership is shown in this plot. (b) Quantities of adopted and rejected plans proposed by each agent. (c) The trajectory of the group plan. (d) The change of the group plan's true utility. Note that agents' attributes are defined in Table 2.

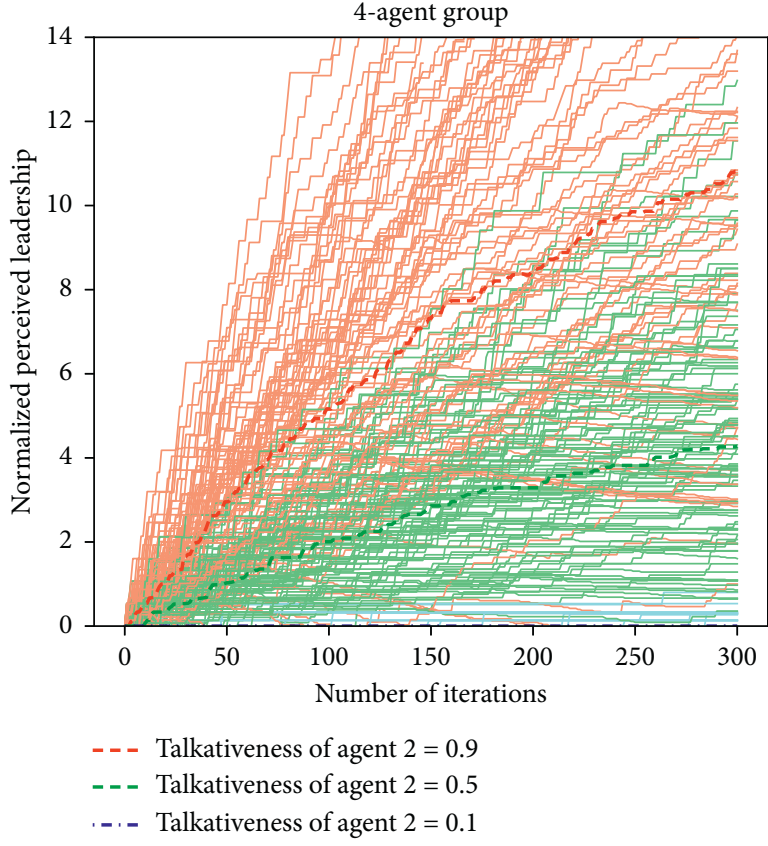

(a)

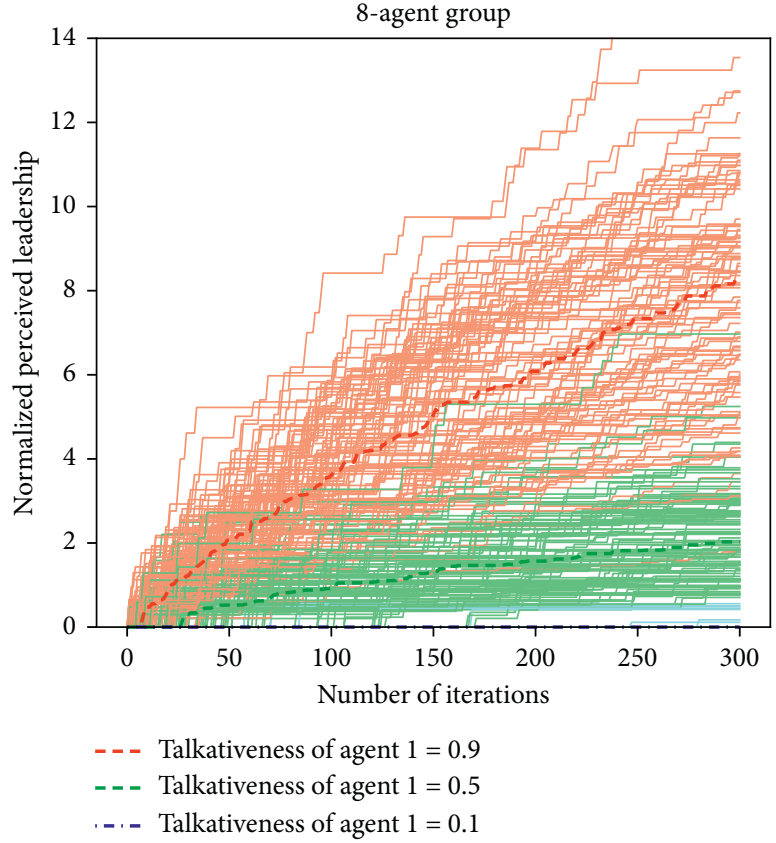

(b)

Figure 5: Perceived leadership of a certain agent (leader) with 3 levels of talkativeness in 4-agent group (a) and 8-agent group (b). The thin lines show the results of 100 independent simulation runs, while the thick dotted lines represent the median of those 100 runs. Note that agents' other attributes are defined in Tables 1 and 2.

The bottom panels of Figure 3 show the change of trajectory (left) and utility (right) of the group plan. The group plan changes drastically with group planning iterations. It could repeat going back and forth between multiple points, which can be seen as the dark blue part in the bottom left panel of Figure 3. In the 4-agent group, the group plan tends to be stable after no more than 100 iterations, which can be seen from the unchanged utility of the group plan in the bottom-right panel of Figure 3.

Simulation results of the 8-agent group are displayed in Figure 4. In the top left panel, from the beginning to the end, agent 1 was the obvious leader in this group because of its high values of talkativeness $\left(T_{1}=0.91\right)$, intelligence $\left(I_{1}=0.95\right)$, and credibility $\left(C_{1}=0.84\right)$. Agent 1 proposed 


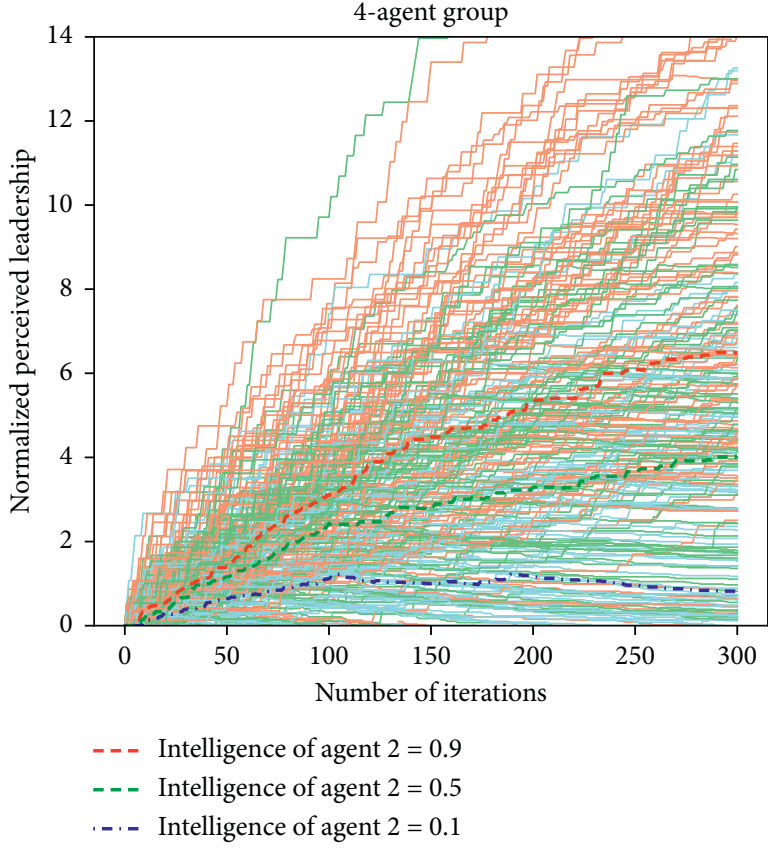

(a)

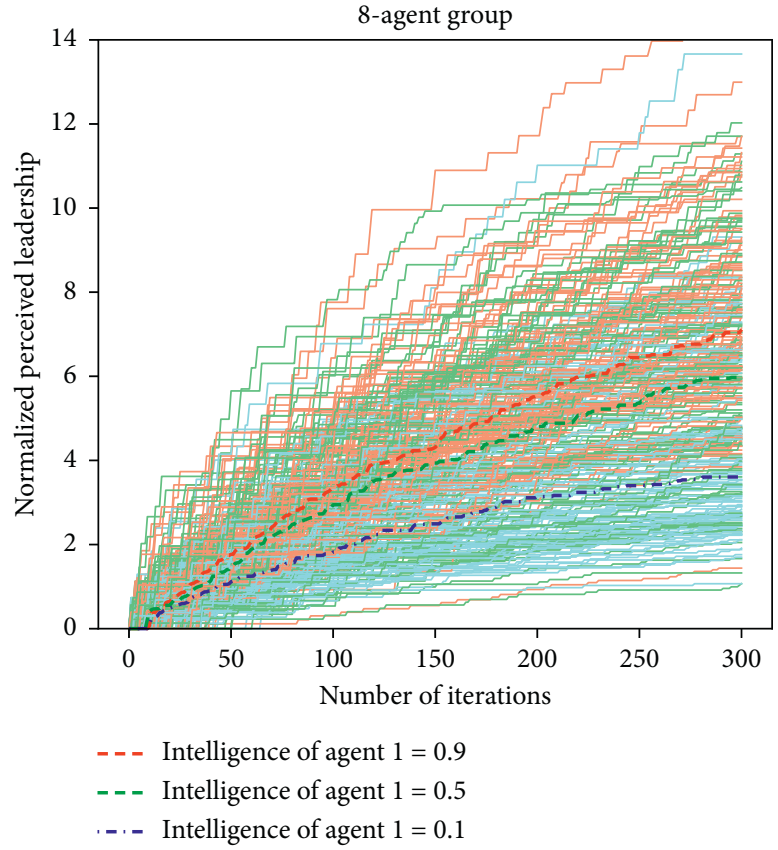

(b)

Figure 6: Perceived leadership of a certain agent (leader) with 3 levels of intelligence in 4-agent group (a) and 8-agent group (b). The thin lines show the results of 100 independent simulation runs, while the thick dotted lines represent the median of those 100 runs. Note that agents' other attributes are defined in Tables 1 and 2.

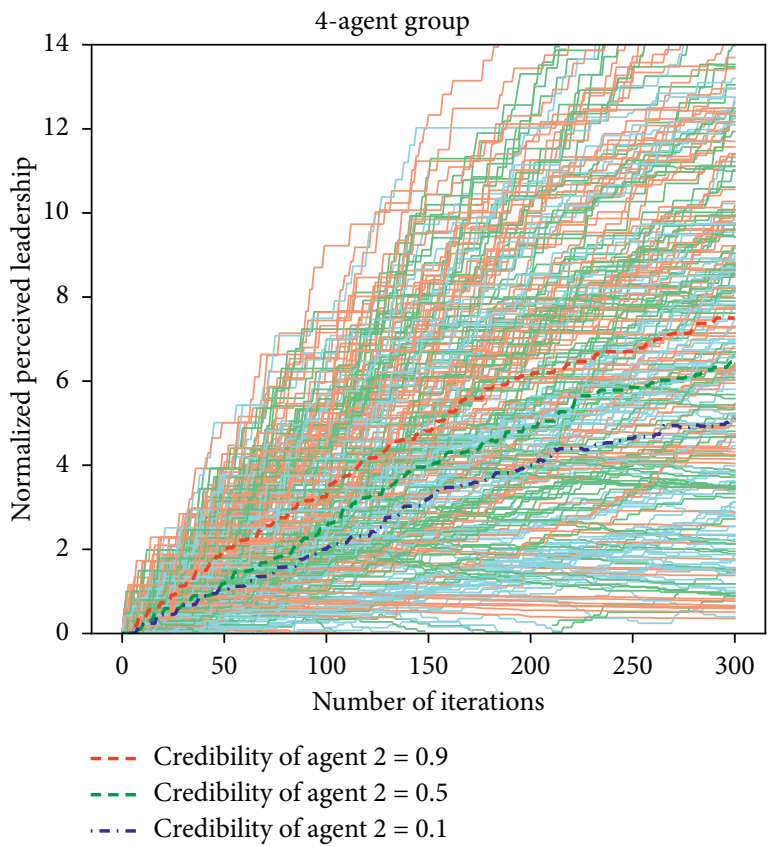

(a)

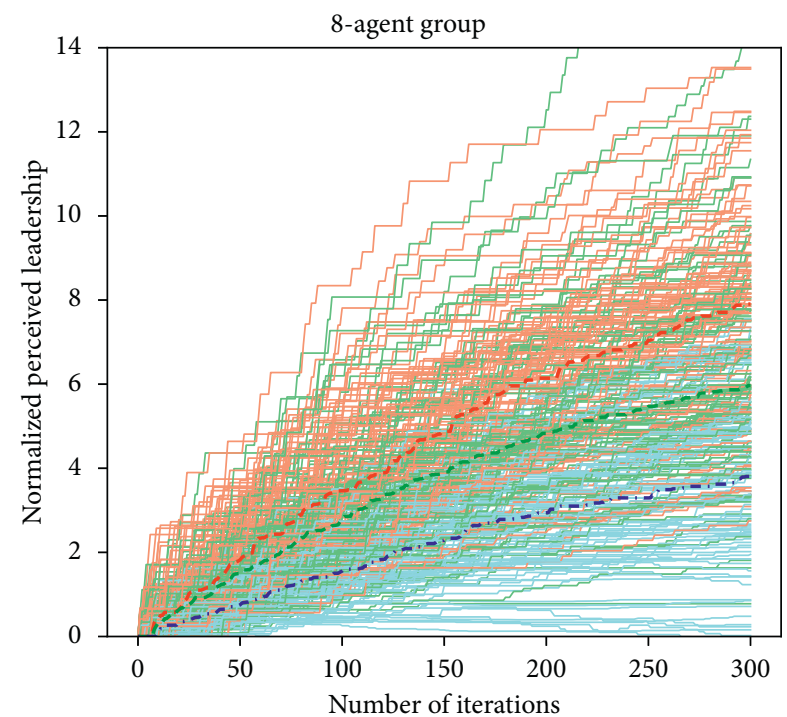

- - - Credibility of agent $1=0.9$

- - - Credibility of agent $1=0.5$

- . . - Credibility of agent $1=0.1$

(b)

Figure 7: Perceived leadership of a certain agent (leader) with 3 levels of credibility in 4-agent group (a) and 8-agent group (b). The thin lines show the results of 100 independent simulation runs, while the thick dotted lines represent the median of those 100 runs. Note that agents' other attributes are defined in Tables 1 and 2.

many suggestions for revising the group plan, most of which were accepted by the group (top-right panel of Figure 4). Meanwhile, both agent 0 and agent 5 did not talk at all in the whole discussion process because of their relatively low talkativeness ( $\mathrm{p})$, and therefore, their perceived leadership remained zero. In contrast, agent 6 talked a lot $\left(T_{6}=0.77\right)$ 


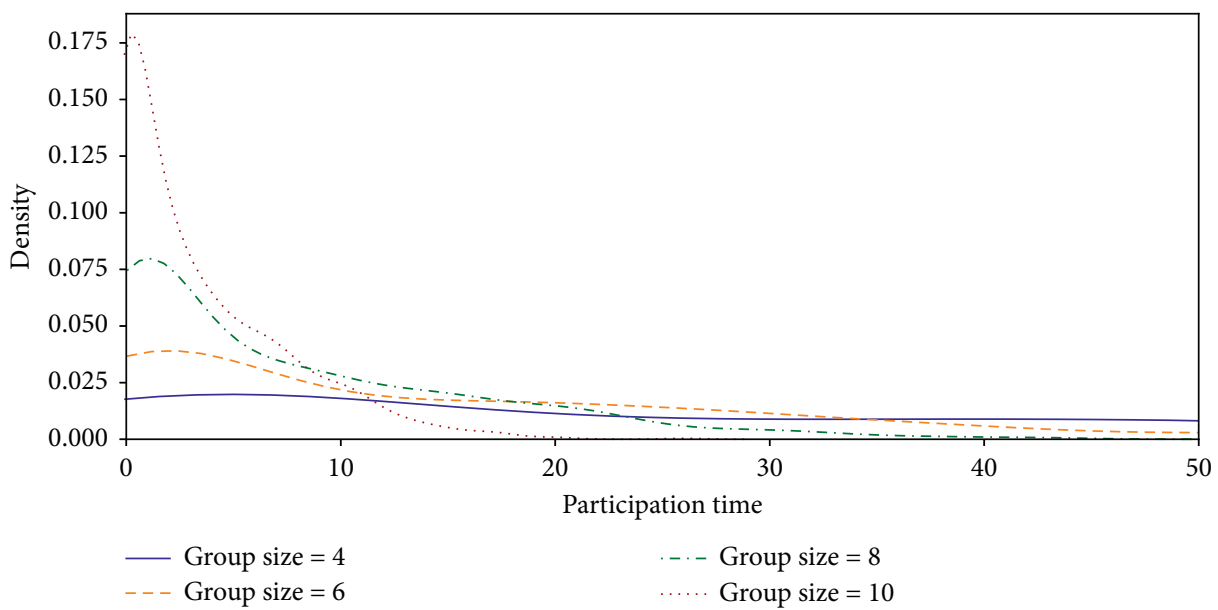

Figure 8: Distribution of participation times in different sized groups. The data was collected from 100 independent simulation runs (300 iterations). The ideal maximal participation time is 300 , yet for a better visualization, here we only show the data within 50 participation times.

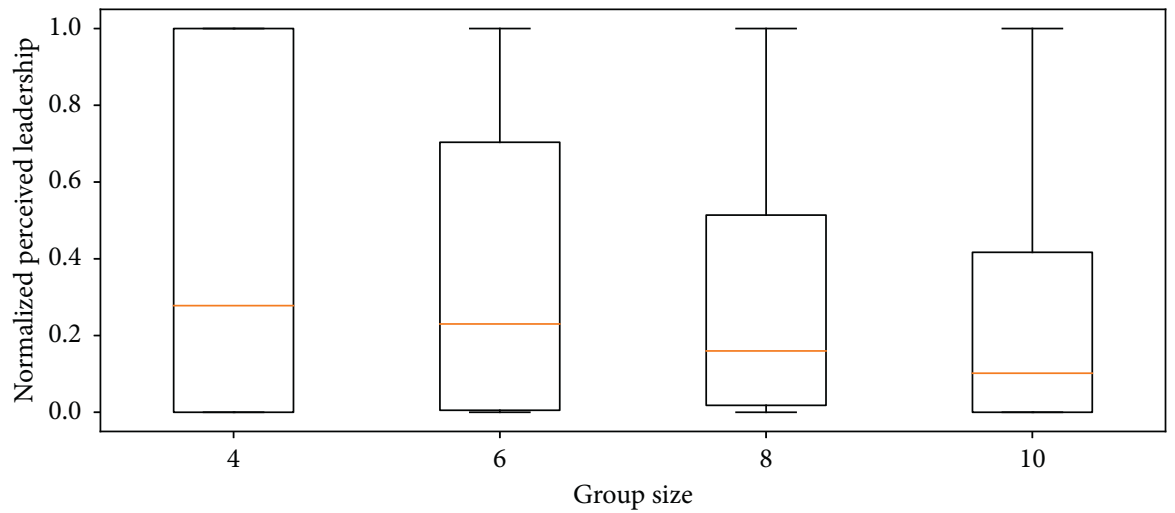

FiguRE 9: Distribution of normalized perceived leadership in different sized groups. Agent $i$ 's normalized perceived leadership after discussion is given by $\left[P L_{i}^{300}-\min \left(P L_{1}^{300}, \ldots, P L_{n}^{300}\right)\right] /\left[\max \left(P L_{1}^{300}, \ldots, P L_{n}^{300}\right)-\min \left(P L_{1}^{300}, \ldots, P L_{n}^{300}\right)\right]$, where $P L_{i}^{300}$ is the perceived leadership of agent $i$ after 300 iterations and $n$ is the size of group.

and proposed many suggestions, but because its intelligence was rather low $\left(I_{6}=0.13\right)$, most of its suggestions were denied by the group (top-right panel of Figure 4). Agents 2, 3,4 , and 7 were not as high as agent 1 regarding the three attributes. Therefore, the perceived leaderships of them are much less than that of agent 1 . The 8 -agent group needed more time to converge than the 4 -agent group. Note that the abovementioned descriptions and explanations are only for the two specific cases shown in Figures 3 and 4.

We also explored talkativeness, intelligence, and credibility one by one to investigate their individual impact on the leader emergence. Here, we chose agent 2 and agent 1 from the representative 4-agent 8-agent groups, respectively, which are defined in Tables 1 and 2, then set three levels $(0.1$, 0.5 , and 0.9 ) of each objective attribute, and ran 100 independent Monte Carlo experiments at each level. All the other model settings remained the same. Simulation results are shown in Figures 5-7. The results revealed that agent's talkativeness, intelligence, and credibility significantly influenced leader emergence. In both 4-agent and 8-agent groups, talkativeness was the most significant predictor for leader emergence since same difference between talkativeness levels could lead more rapidly to much larger variations of perceived leadership than that caused by intelligence or credibility. This observation can be partly corroborated by an empirical study which argued that top talkative people were almost uniformly perceived as contributing more in the experimental groups, whereas the measured intelligence seemed not to be as important as talkativeness [29].

3.2. Collective Leadership Perception in Groups of Different Sizes. We next investigated the impact of group size on the perceived leadership distribution. For the experiments in this section, all the agents' attributes were randomly generated (i.e., the specific illustrative examples of 4 -agent and 8 -agent groups were no longer used). Simulation results are shown in Figures 8 and 9. Figure 8 indicates that the distribution of participation time (speaking time) in larger groups skews more toward zero, which indicates that fewer participants spent a substantial amount of speaking time in the discussion. Figure 9 shows that the average normalized perceived leadership declined significantly as the group size 


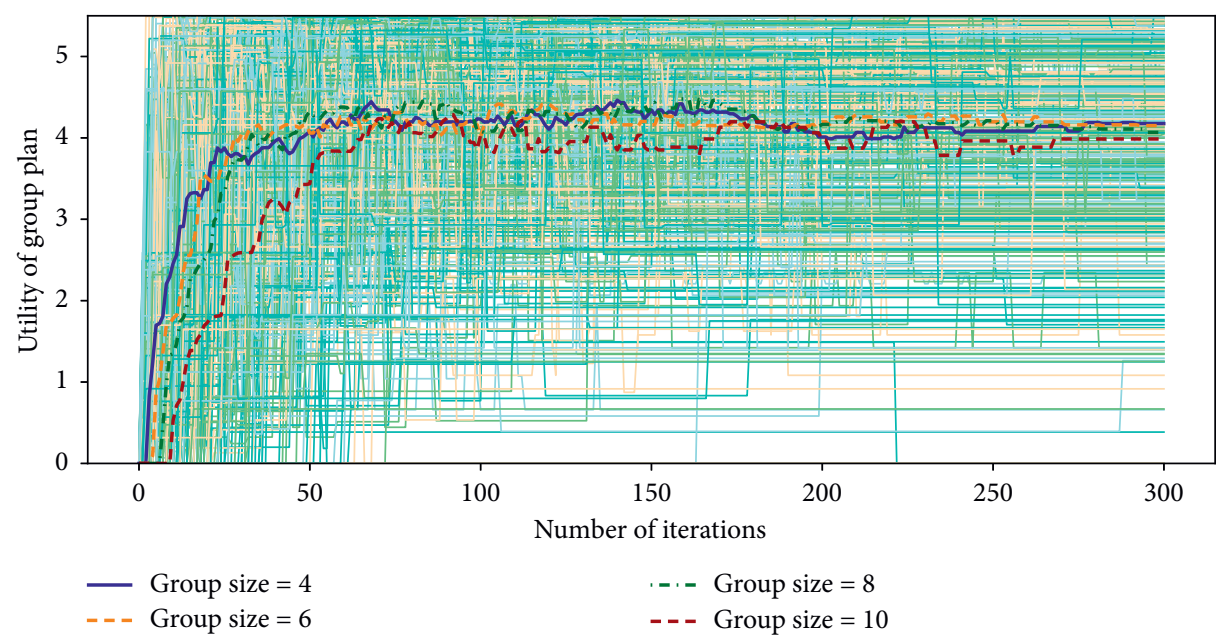

Figure 10: The changes of the utility of group plans during discussion. Thin lines show results from 100 independent runs for each group size, while the thick lines show their medians. Note that we set the initial group plan as $[0,0]$ in the problem space.

increased, indicating the perceived leadership was attributed only to fewer participants. These simulation results could match the empirical studies well $[16,18]$.

3.3. Group Performance of Different Sized Groups. We also investigated the utility of the final group plan obtained in different sized groups. The results are shown in Figure 10, in which two interesting patterns are found. First, smaller sized groups were more likely to outperform larger groups in initial stages of discussion. This is likely because larger groups would need longer time to develop a shared understanding of the problem space. Some studies regarding social organization may bolster our conjecture: members in larger groups feel more anonymous and less involved than they do in smaller groups [30] and the quality of face-to-face communication is worse in larger groups than that in smaller groups [31]. Second, the final impact of group size on the group performance was negligible in the long run. This observation can be understood in which the participation time (and hence the opportunity to contribute) gradually becomes available only to a small number of participants even in larger groups, as shown above. This observation matches the results of the reported works $[18,20]$.

\section{Conclusions}

We studied collective leadership by conducting agent-based simulations of group planning processes. The results offer two interesting observations regarding leader emergence and group performance, which can be summarized as follows:

(1) Talkativeness may be the most significant and instantaneous predictor for leader emergence of the three investigated attributes: talkativeness, intelligence, and credibility

(2) Smaller groups outperform larger groups in the beginning of a group planning process, but their difference tends to become negligible in the long run
These observations match what has been reported in the empirical literature well, indicating the value of our proposed model as a mechanistic, operationalized description of collective leadership and leader emergence.

Our work, however, has several limitations. First, we chose only three individual attributes (i.e., talkativeness, intelligence, and credibility) to construct our model, yet in reality, many other related individual characteristics may also impact human behavior in groups and leader emergence significantly. We plan to include more personal attributes into our model in future work as well as examine the more active role of intelligence and consider the effect of innovation on the results. Second, we assumed that talkativeness, intelligence, and credibility were static individual properties. While reasonable for short-term group planning, this assumption may not be adequate for a model of longer-term team building and leader emergence processes. Third, our investigation is currently limited to small-sized groups (i.e., group size within the range of $4 \sim 10)$. Real-world large-sized groups may typically involve much greater complexity, and therefore, large-sized groups need to be explored further. Fourth, our model is also limited in the ecological validities since we simplified many relationships as simple linear or exponential mathematical expressions in our model which might be otherwise much more complicated in real-world. Finally, the validation of our simulation results with empirical observations has so far remained qualitative only. We plan to conduct more systematic model calibration and fitting so that our model will be more useful for quantitative explanation and prediction of collective leadership processes.

\section{Data Availability}

The simulation model and experimental data used to obtain the findings of this study are available from the corresponding author upon request. 


\section{Disclosure}

The views, opinions, and/or findings contained in this article are those of the authors and should not be construed as an official Department of the Army position, policy, or decision, unless so designated by other documents.

\section{Conflicts of Interest}

The authors declare that there are no conflicts of interest regarding the publication of this paper.

\section{Acknowledgments}

This work was supported by a U.S. Army Research Institute research grant, \#W911NF-17-1-0221.

\section{References}

[1] K. L. Cullen-Lester and F. J. Yammarino, "Collective and network approaches to leadership: special issue introduction," The Leadership Quarterly, vol. 27, no. 2, pp. 173-180, 2016.

[2] T. L. Friedrich, W. B. Vessey, M. J. Schuelke, G. A. Ruark, and M. D. Mumford, "A framework for understanding collective leadership: the selective utilization of leader and team expertise within networks," The Leadership Quarterly, vol. 20, no. 6, pp. 933-958, 2009.

[3] J.-L. Denis, L. Lamothe, and A. Langley, "The dynamics of collective leadership and strategic change in pluralistic organizations," Academy of Management Journal, vol. 44, no. 4, pp. 809-837, 2001.

[4] K. Leithwood and B. Mascall, "Collective leadership effects on student achievement," Educational Administration Quarterly, vol. 44, no. 4, pp. 529-561, 2008.

[5] F. J. Yammarino, E. Salas, A. Serban, K. Shirreffs, and M. L. Shuffler, "Collectivistic leadership approaches: putting the "we" in leadership science and practice," Industrial and Organizational Psychology, vol. 5, no. 4, pp. 382-402, 2012.

[6] K. A. McHugh, F. J. Yammarino, S. D. Dionne, A. Serban, H. Sayama, and S. Chatterjee, "Collective decision making, leadership, and collective intelligence: tests with agent-based simulations and a Field study," The Leadership Quarterly, vol. 27, no. 2, pp. 218-241, 2016.

[7] S. D. Dionne, H. Sayama, C. Hao, and B. J. Bush, "The role of leadership in shared mental model convergence and team performance improvement: an agent-based computational model," The Leadership Quarterly, vol. 21, no. 6, pp. 1035-1049, 2010.

[8] M. S. Mast, "Dominance as expressed and inferred through speaking time: a meta-analysis," Human Communication Research, vol. 28, no. 3, pp. 420-450, 2002.

[9] B. M. Bass, "The leaderless group discussion," Psychological Bulletin, vol. 51, no. 5, pp. 465-492, 1954.

[10] G. E. Littlepage, G. W. Schmidt, E. W. Whisler, and A. G. Frost, "An input-process-output analysis of influence and performance in problem-solving groups," Journal of Personality and Social Psychology, vol. 69, no. 5, pp. 877-889, 1995.

[11] N. G. MacLaren, F. J. Yammarino, S. D. Dionne et al., “Testing the babble hypothesis: speaking time predicts leader emergence in small groups," The Leadership Quarterly, Article ID 101409, 2020.
[12] N. Ensari, R. E. Riggio, J. Christian, and G. Carslaw, "Who emerges as a leader? Meta-analyses of individual differences as predictors of leadership emergence," Personality and Individual Differences, vol. 51, no. 4, pp. 532-536, 2011.

[13] S. J. Zaccaro, J. P. Green, S. Dubrow, and M. Kolze, "Leader individual differences, situational parameters, and leadership outcomes: a comprehensive review and integration," The Leadership Quarterly, vol. 29, no. 1, pp. 2-43, 2018.

[14] R. B. Ruback and J. M. Dabbs, "Talkativeness and verbal aptitude: perception and reality," Bulletin of the Psychonomic Society, vol. 24, no. 6, pp. 423-426, 1986.

[15] B. Z. Posner and J. M. Kouzes, "Relating leadership and credibility,” Psychological Reports, vol. 63, no. 2, pp. 527-530, 1988.

[16] P. D. Reynolds, "Leaders never quit," Small Group Behavior, vol. 15, no. 3, pp. 404-413, 1984.

[17] L. R. Hoffman, "Group problem solving," Advances in Experimental Social Psychology, vol. 2, pp. 99-132, 1965.

[18] B. Mullen, E. Salas, and J. E. Driskell, "Salience, motivation, and artifact as contributions to the relation between participation rate and leadership," Journal of Experimental Social Psychology, vol. 25, no. 6, pp. 545-559, 1989.

[19] J. Antonakis and D. V. Day, Eds., The Nature of Leadership, Sage Publications, Thousand Oaks, CA, USA, 2017.

[20] T. J. Bouchard, J. Barsaloux, and G. Drauden, "Brainstorming procedure, group size, and sex as determinants of the problem-solving effectiveness of groups and individuals," Journal of Applied Psychology, vol. 59, no. 2, pp. 135-138, 1974.

[21] B. S. Bell and S. W. J. Kozlowski, "A typology of virtual teams," Group \& Organization Management, vol. 27, no. 1, pp. 14-49, 2002.

[22] R. T. Keller, "Transformational leadership, initiating structure, and substitutes for leadership: a longitudinal study of research and development project team performance," Journal of Applied Psychology, vol. 91, no. 1, pp. 202-210, 2006.

[23] S. W. J. Kozlowski, "A dynamic theory of leadership and team effectiveness: developmental and task contingent leader roles," Research in Personnel and Human Resources Management, vol. 14, pp. 253-306, 1996.

[24] H. Sayama, Introduction to the Modeling and Analysis of Complex Systems, Open SUNY Textbooks, Albany, NY, USA, 2015.

[25] S. E. Jackson, "The consequences of diversity in multidisciplinary work teams," Handbook of Work Group Psychology, pp. 53-75, John Wiley \& Sons, Chichester, UK, 1996.

[26] R. Likert, "The nature of highly effective groups," Readings in Organizational Behavior, pp. 100-129, McGraw-Hill, New York, NY, USA, 1977.

[27] H. Sayama, D. L. Farrell, and S. D. Dionne, "The effects of mental model formation on group decision making: an agentbased simulation," Complexity, vol. 16, no. 3, pp. 49-57, 2011.

[28] N. S. Altman, "An introduction to kernel and nearestneighbor nonparametric regression," The American Statistician, vol. 46, no. 3, pp. 175-185, 1992.

[29] H. W. Riecken, "The effect of talkativeness on ability to influence group solutions of problems," Sociometry, vol. 21, no. 4, pp. 309-321, 1958.

[30] B. Latan and S. Wolf, "The social impact of majorities and minorities," Psychological Review, vol. 88, no. 5, pp. 438-453, 1981.

[31] P. B. Lowry, T. L. Roberts, N. C. Romano, P. D. Cheney, and R. T. Hightower, "The impact of group size and social presence on small-group communication," Small Group Research, vol. 37, no. 6, pp. 631-661, 2006. 